\title{
Fabricated Antibacterial and Bioactive Titania Nanotube Arrays Coating on the Surface of Titanium
}

\author{
Tian Tian ${ }^{1,2 *}$ Haicheng Dong ${ }^{2}$, Xiaoting Tian², Yafei Hao ${ }^{3}$ \\ ${ }^{1}$ State Key Laboratory of Solidification Processing, Northwestern Polytechnical University, Xi'an, China \\ ${ }^{2}$ No. 52 Institute of China Ordnance Industries, Baotou, China \\ ${ }^{3}$ Erdos Wushen Banner People's Hospital, Erdos, China \\ Email: ${ }^{*}$ fjnutiantian@163.com
}

Received May 30, 2013; revised June 29, 2013; accepted July 13, 2013

Copyright (C) 2013 Tian Tian et al. This is an open access article distributed under the Creative Commons Attribution License, which permits unrestricted use, distribution, and reproduction in any medium, provided the original work is properly cited.

\begin{abstract}
By photic-assisted deposition, $\mathrm{Ag}$ nanoparticles were assembled on bioactive $\mathrm{TiO}_{2}$ nanotube arrays, which were fabricated by anodic oxidation in $0.5 \mathrm{wt} \% \mathrm{NH}_{4} \mathrm{~F}$ solution containing $0.5 \mathrm{wt} \% \mathrm{Na}_{2} \mathrm{HPO}_{4}$. The samples were characterized by scanning electron mincroscope (SEM), X-ray diffraction (XRD). Germiculture experimentation was employed to testing samples' antibacterial capability. An obvious antibacterial ring appeared around the Ag modified samples, indicating that $\mathrm{Ag}$ modified titania nanotube arrays has good antibacterial capability.
\end{abstract}

Keywords: Antibacterial; Bioactive; $\mathrm{TiO}_{2}$ Nanotube Arrays

\section{Introduction}

It is well known that titanium is widely used as bone implant materials because it has excellent biocompatibility and mechanical properties that enhance osseointegration. Nevertheless, it has not bioactive compared to bioactive materials, and requires bio-activation. According to literatures, surface treatments of bioactivities have led to improvements in the adhesion of osteoblasts and their proliferation [1-4]. The surface morphology and composition of the titanium can be regulated by surface treatments, such as alkali and heat treatment, etching, spray, anodizing, and so on.

Branemark et al. [5] reported that titanium and titanium-based alloys can be used as implant materials, many attempts have been made to modify the structure, composition, and chemistry of the titanium surfaces including fabrication of titania coating [6-11], including the fabrication of bioactive $\mathrm{TiO}_{2}$ nanotube arrays $[12,13]$. Tian Tian et al. [12] studied the effect of crystal structure of $\mathrm{TiO}_{2}$ nanotube arrays bioactivity and found that a mixture titania nanotube of anatase and rutile are clearly more efficient in promoting apatite formation than the amorphous state. Xiao Xiufeng et al. [13] found that the bioactive titania nanotube arrays can form on the surface of titanium by anodic oxidation in hydrofluoric (HF) electrolyte with the addition of $5-10 \mathrm{~g} / \mathrm{L} \mathrm{Na}_{2} \mathrm{HPO}_{4}, \mathrm{HPO}_{4}^{2-}$

\footnotetext{
${ }^{*}$ Corresponding author.
}

ions remained in the tube attract $\mathrm{Ca}^{2+}$ in simulated body fluid and induce the nucleation of apatite.

The intuitionistic plot of the titania nanotube arrays is shown in Figure 1(a), the empty space of titania nanotube is very useful for modifying, such as fill something with especial function into it, the intuitionistic plot of modified titania nanotube arrays as shown in Figure 1(b).

Therefore, the present study was aimed to manufacture a new bioactive titanium implant material by modifying bioactive titania nanotube arrays, which will has antibacterial action. Once the bioactive titanium implant material has antibacterial action, they can avoid surgery failing because of infection.

\section{Experiment Section}

\subsection{Preparation of Bioactive Titania Nanotube Arrays}

Prior to anodization, the titanium foils ( $99.5 \%$ pure) were ultrasonically cleaned in acetone and distilled water for 5 $\mathrm{min}$, then chemistry eroded in $4 \mathrm{wt} \% \mathrm{HF}+5 \mathrm{~mol} / \mathrm{L}$ $\mathrm{HNO}_{3}$ for $30 \mathrm{~S}$, followed by ultrasonically cleaned in distilled water for $5 \mathrm{~min}$ and dried in air at 40 of centigrade. A two-electrode with a graphite cathode was employed for the anodization of titanium. $0.5 \mathrm{wt} \% \mathrm{NH}_{4} \mathrm{~F}$ solution counting $5 \mathrm{~g} / \mathrm{L} \mathrm{Na} \mathrm{NPO}_{4}$ solution was used as electrolytes. All electrolytes were prepared from reagent grade 


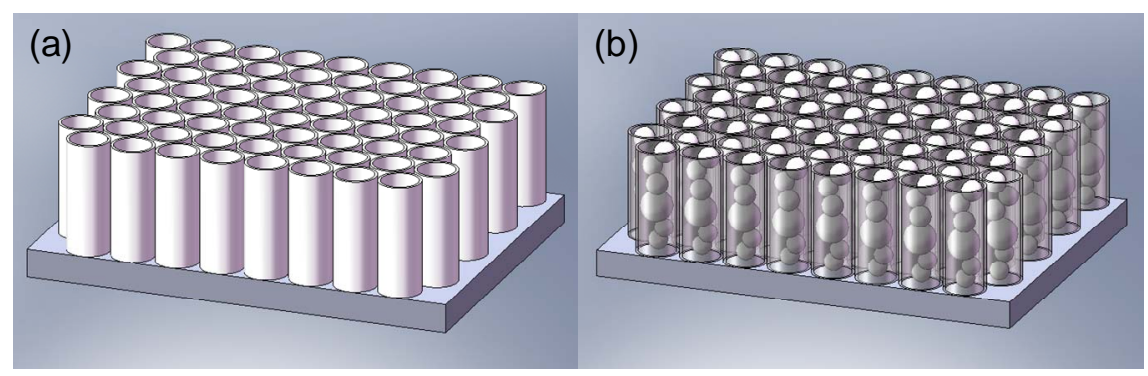

Figure 1. The intuitionistic plot of the titania nanotube arrays before (a) and after (b) modified.

chemicals and DI water. The anodizing voltage was kept constant during the entire process with a DC power supply (GOA, China) at $20 \mathrm{~V}$. The whole course of anodizing was conducted at room temperature ( 25 of centigrade) with magnetic agitation. After anodizing, the samples were rinsed with DI water and then dried at 40 of centigrade in air. The as-prepared sample was signed as NA1.

Heat treated the sample NA1 at $500^{\circ} \mathrm{C}$ in muffle with air for 1hour, and signed it as NA2.

\subsection{Ag Modified Bioactive Titania Nanotube Arrays}

After immerging the samples into $0.1 \mathrm{~mol} \cdot \mathrm{L}^{-1} \mathrm{AgNO}_{3}$ solutions for $2 \mathrm{~min}$, irradiated the samples by ZF-II UV lamp with the wavelengh of $365 \mathrm{~nm}$ and the intensity of $1400 \mathrm{mw} \cdot \mathrm{cm}^{-2}$ for $2 \mathrm{~min}$. Repeat the process three times. At last ultrasonically cleaned the samples with DI water and dried them at $40^{\circ} \mathrm{C}$ in air, and signed the sample as NA3.

\subsection{Antibacterial Researches}

Staphyloccocus aureus is an important pathogeny bacterium for human. It can evoke many badly infection. So the staphyloccocus aureus was used in the antibacterial researches.

Firstly, coated the MH agar plate onto the petri dish, and then coated the asepsis physiological brine with 0.5 $\mathrm{wt} \%$ staphyloccocus aureus onto the $\mathrm{MH}$ agar plate, ultimately put the petri dish into the electrothermal incubator with $37^{\circ} \mathrm{C}$ temperature for 12 hours and 24 hours. But 12 hours is not enough for antibacterial researches to make a distinction. Therefore longer time spend on the antibacterial researches in this section.

\subsection{Characterization}

Scanning electron microscopy (SEM) was used to determine surface morphology using HITACHI-S3400N SEM. The compositions of the coating were determined with an energy dispersive X-ray spectrometer (EDX).

\section{Results and Discussions}

From Figure 2, we can see the mouth of titania nano-

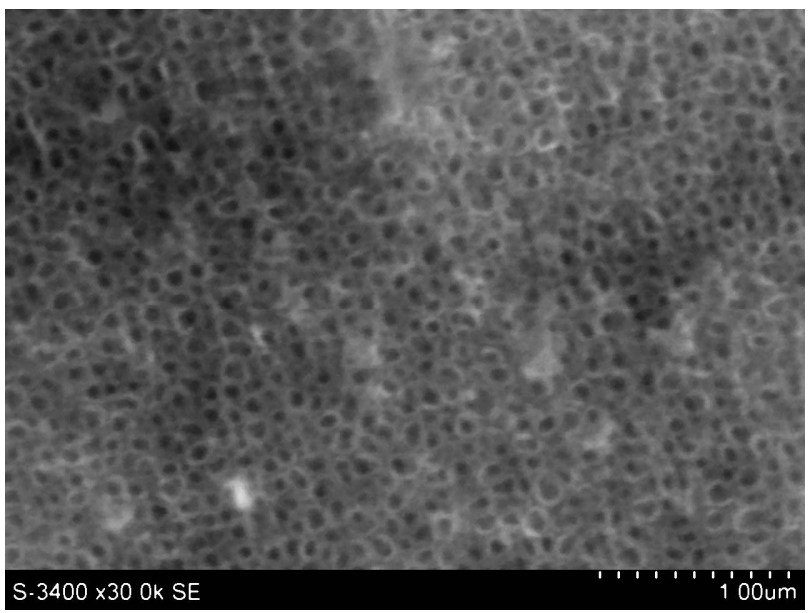

Figure 2. Surface morphology of NA3.

tube. When the sample NA3 was tested by XRD, Ti peak and $\mathrm{TiO}_{2}$ peak appeared but there is no Ag peak on the XRD patterns. The result of XRD is shown in Figure 3 XRD of NA3.

The results indicated that the amount of $\mathrm{Ag}$ is little. The compositions of the coating were determined with EDX, the result as shown in Figure 4. There has about $2.3 \mathrm{wt} \% \mathrm{Ag}$ formed on the surface of the sample.

The results of the EDX and the real reaction as follow can prove effectively that $\mathrm{Ag}$ have formed on the surface of NA3. The results indicated that Ag modified was successful.

$$
2 \mathrm{AgNO}_{3}+\mathrm{hv}=2 \mathrm{Ag} \downarrow+2 \mathrm{NO}_{2} \uparrow+\mathrm{O}_{2} \uparrow
$$

In antibacterial researches, there is an obviously antibacterial ring formed around the NA3, and there is no antibacterial ring formed around the NA1 and NA2 as contrast. The result is shown in Figure 5, the result of antibacterial researches. The results indicate that Ag modified is successful, the above process endow the bioactive titania nanotube arrays with good antibacterial capability.

It is well known that Ag has good antibacterial capability. Modifying the bioactive titania nanotube arrays with $\mathrm{Ag}$ is a good idea for the fabrication of the antibacterial bioactive titania nanotube arrays. 


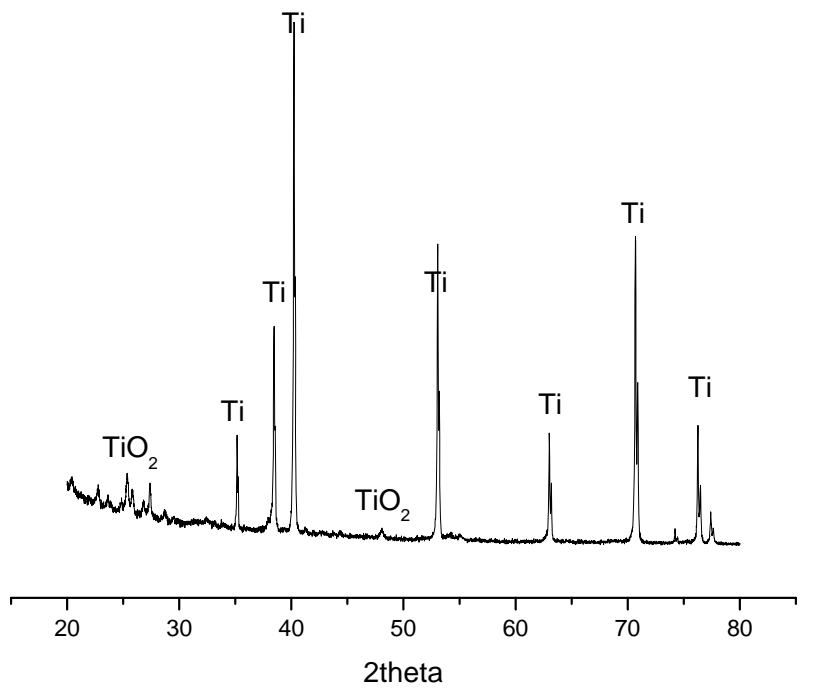

Figure 3. XRD of NA3.

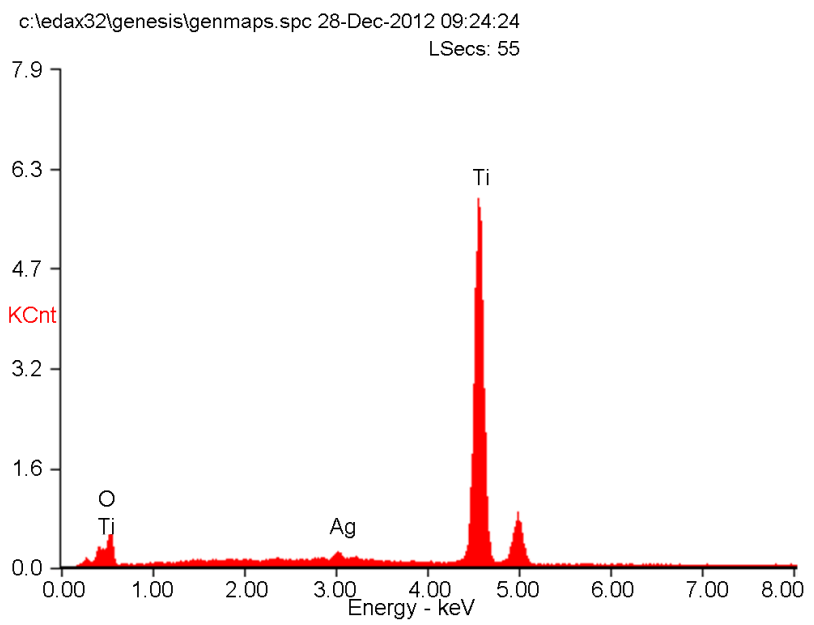

Figure 4. EDX of NA3.

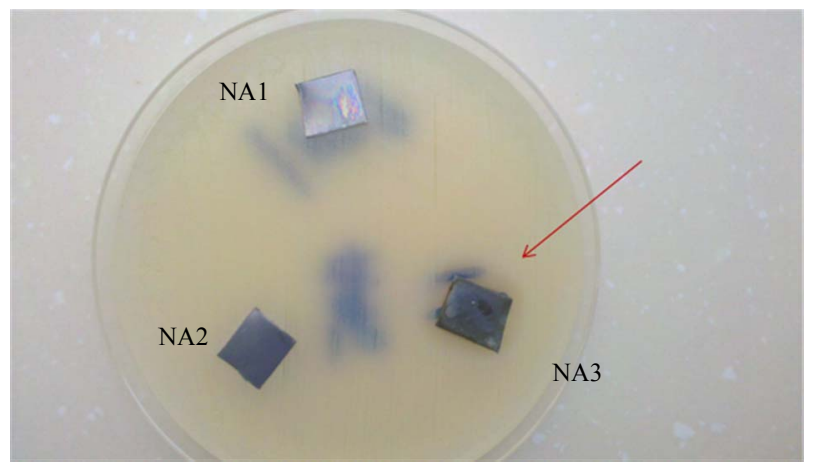

Figure 5. The result of antibacterial researches.

\section{Conclusion}

The antibacterial bioactive titania nanotube arrays can be obtained by follow method: modify the bioactive titania nanotube arrays with Ag. Anodic oxidation treatment and Ag modified are economical method for surface an- tibacterial bioactive treatment.

\section{Acknowledgements}

The authors would like to thanks the Research Foundation of Baotou City Youth Innovation and the Research Foundation of Zhejiang Province Public Technology Research and industrial projects (2013C31051).

\section{REFERENCES}

[1] Z. Schwartz, K. Kieswetter, D. D. Dean and B. D. Boyan, "Underlying Mechanism at the Bone-Surface Interface during Regeneration," Journal of Periodontal Research, Vol. 32, No. 1, 1997, pp. 166-171. doi:10.1111/j.1600-0765.1997.tb01399.x

[2] B. Chehroudi, D. McDonnel and D. M. Brunette, "The Effects of Micromachined Surfaces on Formation of Bonelike Tissue on Subcutaneous Implants as Assessed by Radiography and Computer Image Processing," Journal of Biomedical Materials Research, Vol. 34, No. 3, 1997, pp. 279-290.

doi:10.1002/(SICI)1097-4636(19970305)34:3<279::AIDJBM2>3.0.CO;2-H

[3] X. Zhu, J. Chen, L. Scheideler, T. Altebaeumer and J. GeisGerstorfer, "Cellular Reactions of Osteoblasts to Micron and Submicron-Scale Porous Structures of titanium Surfaces," Cells Tissues Organs, Vol. 178, 2004, pp. 1322. doi:10.1159/000081089

[4] G. E. II Aninwene, C. Yao and T. J. Webster, "Enhanced Osteoblast Adhesion to Drug-Coated Anodized Nanotubular Titanium Surfaces," International Journal of Nanomedicine, Vol. 3, No. 2, 2008, pp. 257-264.

[5] P. I. Branemark, "Osseointegrated Implants in the Treatment of the Edentulous Jaw. Experience from a 10-Year Period," Journal of Plastic and Reconstructive Surgery, Vol. S16, 1977, pp. 1-132.

[6] P. Babelon, A. S. Dequiedt, H. Moste'fa-Sba, S. Bourgeois, P. Sibillot and M. Sacilotti, "SEM and XPS Studies of Titanium Dioxide Thin Films Grown by MOCVD," Thin Solid Films, Vol. 322, No. 1-2, 1998, pp. 63-67. doi:10.1016/S0040-6090(97)00958-9

[7] S. Thybo, S. Jensen, J. Johansen, T. Johannessen, O. Hansen and U. J. Quaade, "Flame Spray Deposition of Porous Catalysts on Surfaces and in Microsystems," Journal of Catalysis, Vol. 223, No. 2, 2004, pp. 271-277. doi:10.1016/j.jcat.2004.01.027

[8] N. N. Dinh, N. T. T. Oanh, P. D. Long, M. C. Bernard and A. H. Goff, "Electrochromic Properties of $\mathrm{TiO}_{2}$ Anatase Thin Films Prepared by a Dipping Sol-Gel Method," Thin Solid Films, Vol. 423, No. 1, 2003, pp. 70-76. doi:10.1016/S0040-6090(02)00948-3

[9] C. Lee, H. Choi, C. Lee and H. Kim, "Photocatalytic Properties of Nano-Structured $\mathrm{TiO}_{2}$ Plasma Sprayed Coating," Surface Coat Technology, Vol. 173, No. 2-3, 2003, pp. 192-200.

[10] T. Sumita, T. Yamaki, S. Yamamoto and A. Miyashita, "Photo-Induced Surface Charge Separation in Cr-Implanted $\mathrm{TiO}_{2}$ Thin Films," Thin Solid Films, Vol. 416, No. 1-2, 
2002, pp. 80-84. doi:10.1016/S0040-6090(02)00618-1

[11] H. Ohsaki, Y. Tachibana, A. Mitsui, T. Kamiyama and Y.

[12] Hayashi, "High Rate Deposition of $\mathrm{TiO}_{2}$ by DC Sputtering of the $\mathrm{TiO}_{2-\mathrm{x}}$ Target," Thin Solid Films, Vol. 392, No. 2, 2001, pp. 169-173. doi:10.1016/S0040-6090(01)01023-9

[13] T. Tian, X. Xiufeng, S. Houde and L. Rongfang, "Biomimetic Growth of Apatite on Titania Nanotube Arrays Fabricated by Titanium Anodization in $\mathrm{NH}_{4} \mathrm{~F} / \mathrm{H}_{2} \mathrm{SO}_{4}$
Electrolyte," Materials Science-Poland, Vol. 26, No. 3, 2008, pp. 487-494.

[14] X. Xiufeng, L. Rongfang and T. Tian, "Preparation of Bioactive Titania Nanotube Arrays in $\mathrm{HF} / \mathrm{Na}_{2} \mathrm{HPO}_{4}$ Electrolyte," Journal of Alloys and Compounds, Vol. 466, No. $1-2,2008$, pp. 356-362. 